Received: 26.04 .2018

Revised: 15.10 .2018

Accepted: 26.10 .2018

DOI: $10.17804 / 2410-9908.2018 .6 .191-202$

\title{
MODELING OF THE MULTI-LEVEL PROCESS OF DAMAGE ACCUMULATION IN A REFLECTOR SKELETON WITH AN INHOMOGENEOUS STRUCTURE
}

\author{
S. V. Doronin ${ }^{\text {a)* }}$ and Yu. F. Filippova ${ }^{\text {b) }}$ \\ Institute of Computational Technologies, SB RAS, Krasnoyarsk Branch Office, \\ 53 Mira Ave., 660049, Krasnoyarsk, Russian Federation \\ a) (iD https://orcid.org/0000-0002-5256-3871 mr.svdoronin@yandex.ru; \\ b) iD https://orcid.org/0000-0001-7452-2096冈 filippovajf@ict.nsc.ru \\ *Corresponding author. E-mail: filippovajf@ict.nsc.ru \\ Address for correspondence: P.O. Box No. 25515, Krasnoyarsk, 660049, Russian Federation \\ Tel.: +7 (391) 2277296
}

An information-computing model for analyzing the damage and survivability of the joint assembly of a beam skeleton of a large parabolic antenna reflector is considered. The joint assembly integrates composite pipes and fittings by means of bolting and glue joints. The informationcomputing model is described by frames, that is, the structures of the data of the system components and relations between them. The model involves numerical analysis of damage and failure.

Keywords: reflector skeleton, joint assembly, bolted joint, adhesive layer, scenario, damage accumulation, survivability, information model.

\section{References}

1. Frolov K.V. Kudinov V.M., Makhutov N.A. Applications of Mechanics to Technological Problems (Mechanical Engineering \& Applied Mechanics), CRC Press, 1999, 410 p.

2. $\quad$ Case J., Chilver A.H., Ross C.T.F. Strength of Materials and Structures, 4th edition, Butterworth-Heinemann, 1999, $724 \mathrm{p}$.

3. Jenkins C.H.M. Mechanics of materials: A Modern Integration of Mechanics and Materials in Structural Design, Elsevier Academic Press, 2005, 408 p.

4. Parnes R. Solid Mechanics in Engineering, first edition, John Wiley \& Sons, 2001, 748 p.

5. Popov E.P. Engineering Mechanics of Solids, second edition, Prentice Hall of India, 1998, $888 \mathrm{p}$.

6. Gol'denblat I.I. Some Problems of the Mechanics of Deformable Media, Noordhoff, 1962, $304 \mathrm{p}$.

7. Perelmuter A.V. On progressive failure calculations. Vestnik MGSU, 2008, no. 1, pp. 119-129. (In Russian).

8. Kolchunov, V.I., Klueva, N.V., Androsova, N.B., Bukhtiyarova, A.S. Zhivuchest zdaniy i sooruzheniy pri zaproyektnykh vozdeystviyakh [Survivability of buildings and structures beyond design actions]. Moscow, ASV Publ., 2014, 208 p. (In Russian).

9. Gordon V.A., Klyuyeva N.V., Poturayeva T.V., Bukhtiyarova A.S. Calculation of dynamic forces in structurally nonlinear elements of spatial rod systems under sudden structural changes. Stroitelnaya Mekhanika i Raschet Sooruzheniy, 2008, no. 6, pp. 26-30. (In Russian).

10. Makhutov N.A., Reznikov D.O., Petrov P.V. Assessment of survivability of complex technical systems. Problemy Bezopasnosti i Chrezvychaynykh Situatsiy, 2009, no. 3. pp. 47-66. (In Russian).

11. Bathe K.-J. Finite element procedures, Prentice-Hall, New Jersey, 1996, 1038 p. 
12. Wriggers P. Computational contact mechanics, Berlin-Helderberg, Springer, 2006, 521 p. 13. Komarov G.V. PKM properties affecting their ability to connect. Polimernyye materialy, 2010, nos. 2-3, pp. 18-27. (In Russian).

14. Dveyrin A.Z., Krivenda S.P. Testing of laminated plastics for crushing. In: Voprosy Proektirovaniya i Proizvodstva Konstruktsiy Letatelnykh Apparatov: sb. nauch. trudov. nats. aerokosm. un-ta im. N.E. Zhukovskogo «KhAI», 2011, no. 1 (65), pp. 20-28. (In Russian).

15. Semin M.I. Raschety soyedineniy elementov konstruktsiy iz polimernykh materialov na prochnost $i$ dolgovechnost [Calculations of Joints of Structural Elements Made of Polymer Materials for Strength and Durability]. Moscow, MADI Publ., 2016, 92 p. (In Russian). 
Подана в журнал: 26.04.2018

УДК $621.81: 539.42$

DOI: $10.17804 / 2410-9908.2018 .6 .191-202$

\title{
МОДЕЛИРОВАНИЕ МНОГОУРОВНЕВОГО ПРОЦЕССА НАКОПЛЕНИЯ ПОВРЕЖДЕНИЙ СТРУКТУРНО-НЕОДНОРОДНОГО КАРКАСА РЕФЛЕКТОРА
}

\author{
С. В. Доронин ${ }^{\mathrm{a})^{*}}$, Ю. Ф. Филиппова ${ }^{\text {б) }}$ \\ Институт вычислительных технологий СО РАН, Красноярский филиал, \\ пр. Мира, 53, г. Красноярск, 660049, Российская Федераџия \\ a) iD https://orcid.org/0000-0002-5256-3871 mr.svdoronin@yandex.ru; \\ б) (iD https://orcid.org/0000-0001-7452-2096 $\otimes$ filippovajf@ict.nsc.ru \\ *Ответственный автор. Электронная почта filippovajf@ict.nsc.ru \\ Адрес для переписки: 660049, а/я 25515, Красноярск, Российская Федерация \\ Тел.: +7 (391) 227-72-96
}

В работе рассматривается информационная и вычислительная модель анализа повреждений и живучести узла сочленения стержневого каркаса крупногабаритного рефлектора параболической зеркальной антенны. Узел сочленения представляет собой соединение композитных труб и фитингов посредством болтовых и клеевого соединений. Информационная и вычислительная модель описывается фреймами - структурами данных элементов системы и связей между ними и основана на использовании численного анализа повреждений и разрушений.

Ключевые слова: каркас рефлектора, узел сочленения, болтовое соединение, клеевой слой, сценарий, накопление повреждений, живучесть, информационная модель.

\section{1. Введение}

Классические подходы к инженерным расчетам структурно и геометрически сложных конструкций основаны на идее их декомпозиции. В этом случае конструкцию мысленно расчленяют на отдельные относительно простые части (узлы, детали), каждую из которых рассчитывают по отдельности, а влияние остальных частей учитывают комплексом механических взаимодействий. Развитие методов механики материалов, накопление экспериментальных данных о механических свойствах широкого спектра конструкционных материалов привело к тому, что инженерное проектирование обеспечено большим количеством выраженных в общем виде уравнений предельных состояний и вытекающих из них неравенств - условий прочности [1-6]. Эти условия представляют собой методическое и информационное обеспечение расчета отдельных частей, выделенных из конструкции в результате декомпозиции.

Точность результатов, полученных на основе такого подхода, неопределена. Для каждой конструкции различные варианты декомпозиции характеризуются собственным перечнем упрощений и допущений. Очевидна тенденция: по мере увеличения габаритов, геометрической и структурной сложности конструкций, усложнения их взаимодействия с системным окружением точность расчетов, основанных на декомпозиции конструкций, в общем случае снижается. Кроме того, применение указанных условий прочности часто связано с затруднениями определения входящих в неравенства величин и параметров.

Развитие методов математического и вычислительного моделирования, численных методов анализа (преимущественно методов конечных элементов), программных и аппаратных средств вычислительной техники открыло принципиальную возможность выполнять расчет достаточно сложных конструкций как единого целого. Это способствовало суще- 
ственному прогрессу инженерных расчетов на стадии проектирования конструкций, повышению их качественных характеристик.

Однако остаются слабо изученными вопросы прогнозирования поведения конструкций на стадии эксплуатации, характеризующейся возникновением, накоплением, развитием повреждений отдельных конструктивных элементов и сборки в целом. С одной стороны, проверка условий прочности для отдельных деталей и элементов конструкций не позволяет судить о поведении конструкции при нарушении одного или нескольких условий прочности, ее способности сохранять ограниченную во времени или по условиям нагружения работоспособность. С другой, - не вполне проработаны методические вопросы моделирования повреждаемых конструкций. Исследования в этой области носят преимущественно академический или объектно-ориентированный характер [7-10] и в малой степени могут быть применены к решению инженерных задач для произвольных конструкций.

В связи с этим актуальна разработка методического обеспечения анализа живучести конструкций, повреждения которых развиваются во временной последовательности и причинно-следственной зависимости. Она осуществляется на базе междисциплинарного подхода, объединяющего методы механики материалов, вычислительной математики и механики, системного анализа, инженерии знаний, и ориентирована на предметную область конструкторско-технологической информатики и использование в технологиях информационной поддержки жизненного цикла. Апробация подходов выполнена для узла сочленения стержневого каркаса крупногабаритного рефлектора параболической зеркальной антенны.

\section{2. Постановка задачи и методы решения}

Объектом исследования является крупногабаритный прецизионный рефлектор зеркальной антенны наземных систем спутниковой связи $Q / K_{a}$-частотного диапазона (рис. 1 ). Диаметр рефлектора составляет 12 м. Каркас представляет собой пространственную стержневую конструкцию (рис. 2). Стержни представлены трубами квадратного поперечного сечения 60×60 мм. Используется два варианта толщины стенки труб: на периферии $-1,4$ мм, в области ступицы - 2,8 мм. Соединение труб осуществляется с использованием фитингов (толщина стенки 2,8 мм) посредством болтовых и клеевого соединений.

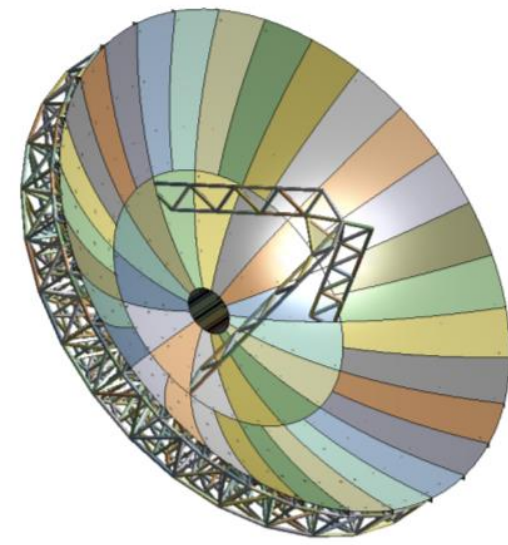

$a$

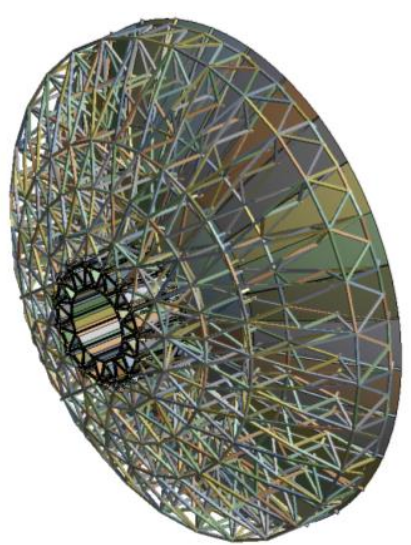

$\sigma$

Рис. 1. Общий вид рефлектора 


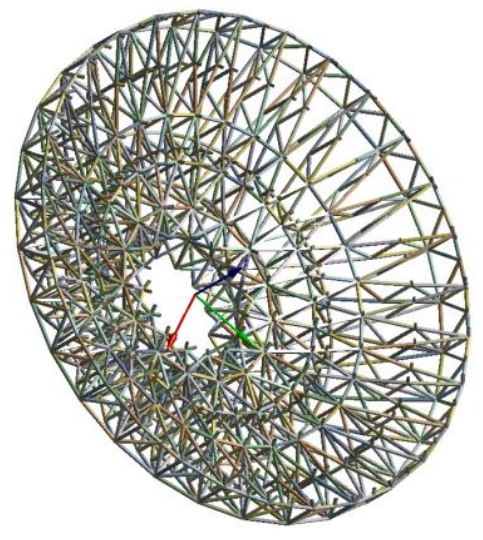

$a$

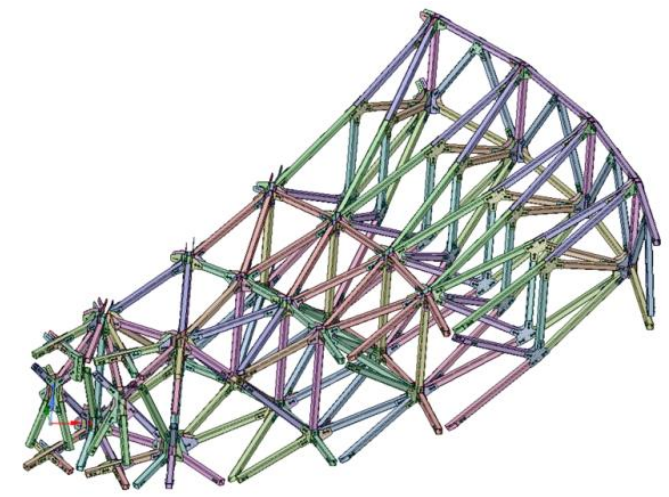

$\sigma$

Рис. 2. Строение каркаса рефлектора

Каркас характеризуется высокой степенью структурной неоднородности, обусловленной тем, что

- используются трубы различной длины и различного поперечного сечения;

- узлы сочленения стержней имеют различное конструктивное оформление в зависимости от количества входящих в узел стержней (рис. 3);

- используются различные варианты однонаправленных и тканых композитов, описываемых моделями ортотропных материалов;

- используются композиты и стальные детали - неоднородность вследствие существенно различной жесткости;

- присутствует трение, преднатяжение болтов, клеевое соединение - неоднородность физико-технических процессов в соединениях деталей.

Наличие указанных неоднородностей является определяющим фактором в выстраивании технологии анализа повреждаемости каркаса рефлектора, разработки его информационного и методического обеспечения.

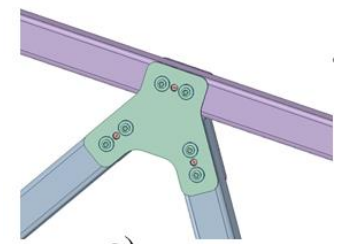

$a$

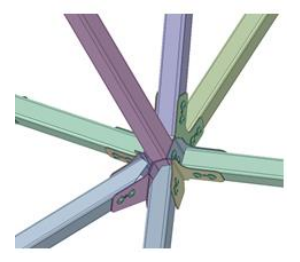

$\partial$

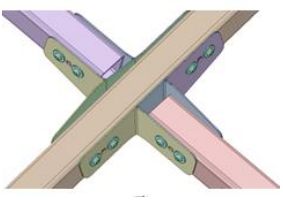

6

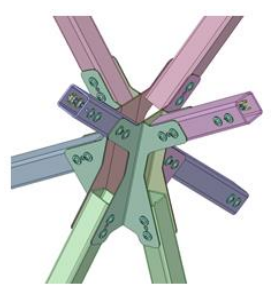

$e$

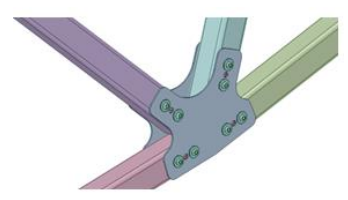

B

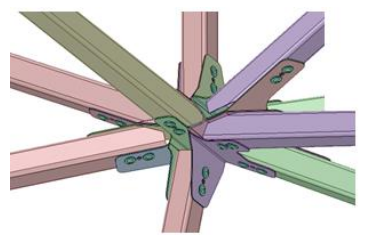

Ж

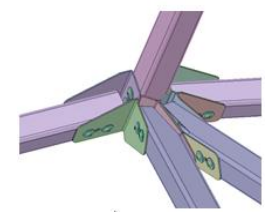

2

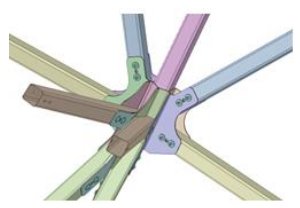

3

Рис. 3. Варианты узлов сочленения стержней каркаса с использованием фитингов: $a, \sigma-3$ стержня; $6-4$ стержня; $2-5$ стержней; $\partial-7$ стержней; $e-8$ стержней; ж, з - 9 стержней 
Цель исследования - разработка и апробация информационно-вычислительной технологии моделирования развития и накопления повреждений каркаса рефлектора в их временной и причинно-следственной взаимосвязи и взаимообусловленности.

Для достижения этой цели сформулированы следующие задачи:

1) выстраивание процедуры построения структурной и информационной моделей конструкций;

2) обоснование методического подхода к построению информационной модели живучести, учитывающей структурные взаимодействия элементов конструкции, взаимосвязь и взаимообусловленность процессов накопления повреждений;

3) разработка и апробация человеко-машинной процедуры анализа живучести повреждаемой конструкции.

Процедура построения структурной и информационной моделей конструкции заключается в анализе совместной работы элементов конструкции и их системных взаимосвязей при работе, как в штатном, так и в поврежденном состоянии. Для конкретизации рассмотрим элементарный, повторяющийся фрагмент узла сочленения стержней каркаса. Он включает трубу и фитинг из полимерных композиционных материалов различной структуры, стальные обойму, болты и гайки (рис. 4). Несмотря на небольшое число конструкционных элементов, объединение их в систему осуществляется с помощью разнородных физико-технических связей: резьбовых и клеевых соединений, фрикционных взаимодействий. Это формирует высокую степень структурной неоднородности конструкции.
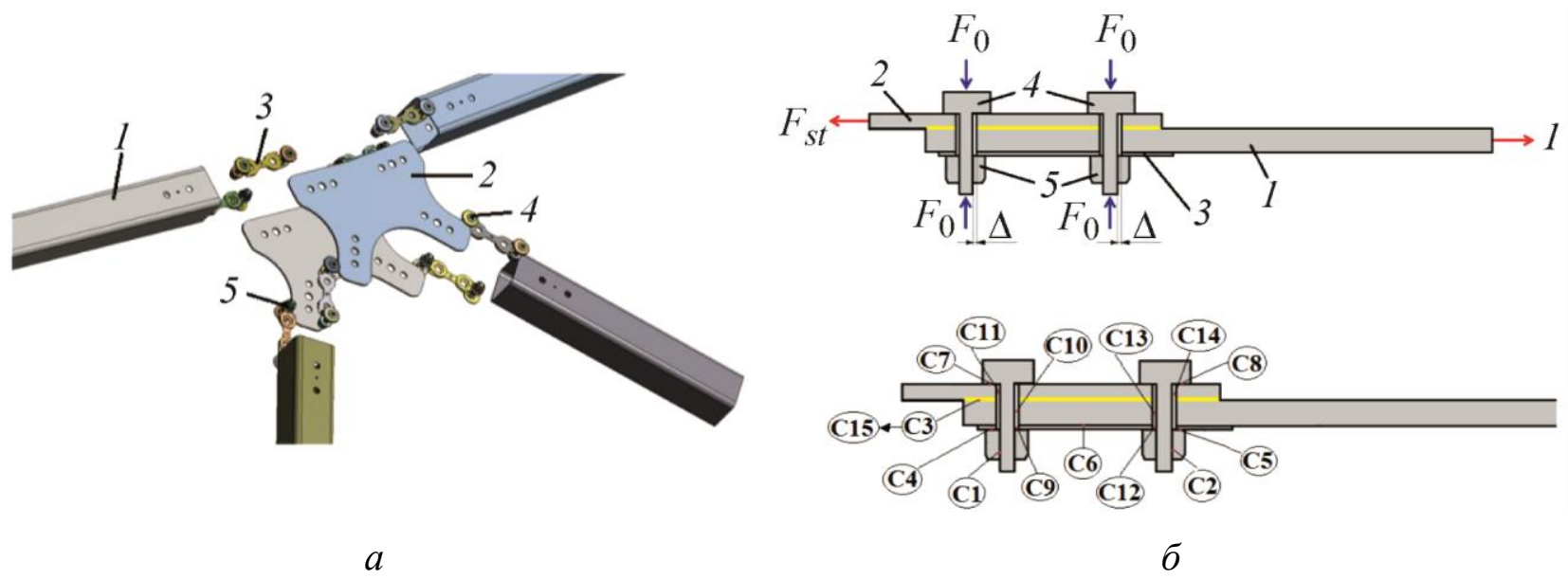

б

Рис. 4. Структурная схема (модель) узла сочленения трубы с фитингом (рис. 3 в): 1 - труба; 2 - фитинг; 3 - обойма; 4 - болт; 5 - гайка; С1, С2 - резьбовые соединения; С3 - клеевое соединение; C4-C15 - фрикционные взаимодействия; $\Delta$ - зазор

Информационная модель структуры и конструкционной прочности узла включает два компонента: 1 - информационные модели элементов конструкции и их связей; 2 общие формулировки всех условий прочности, определяющих конструкционную целостность узла.

Свойства поименованных элементов и связей описываются фреймами - структурами данных, однозначно идентифицирующими объект. Совокупность элементов конструкции, их связей и фрейм-описаний характеристик представляет собой информационную модель конструкции (рис. 5). 


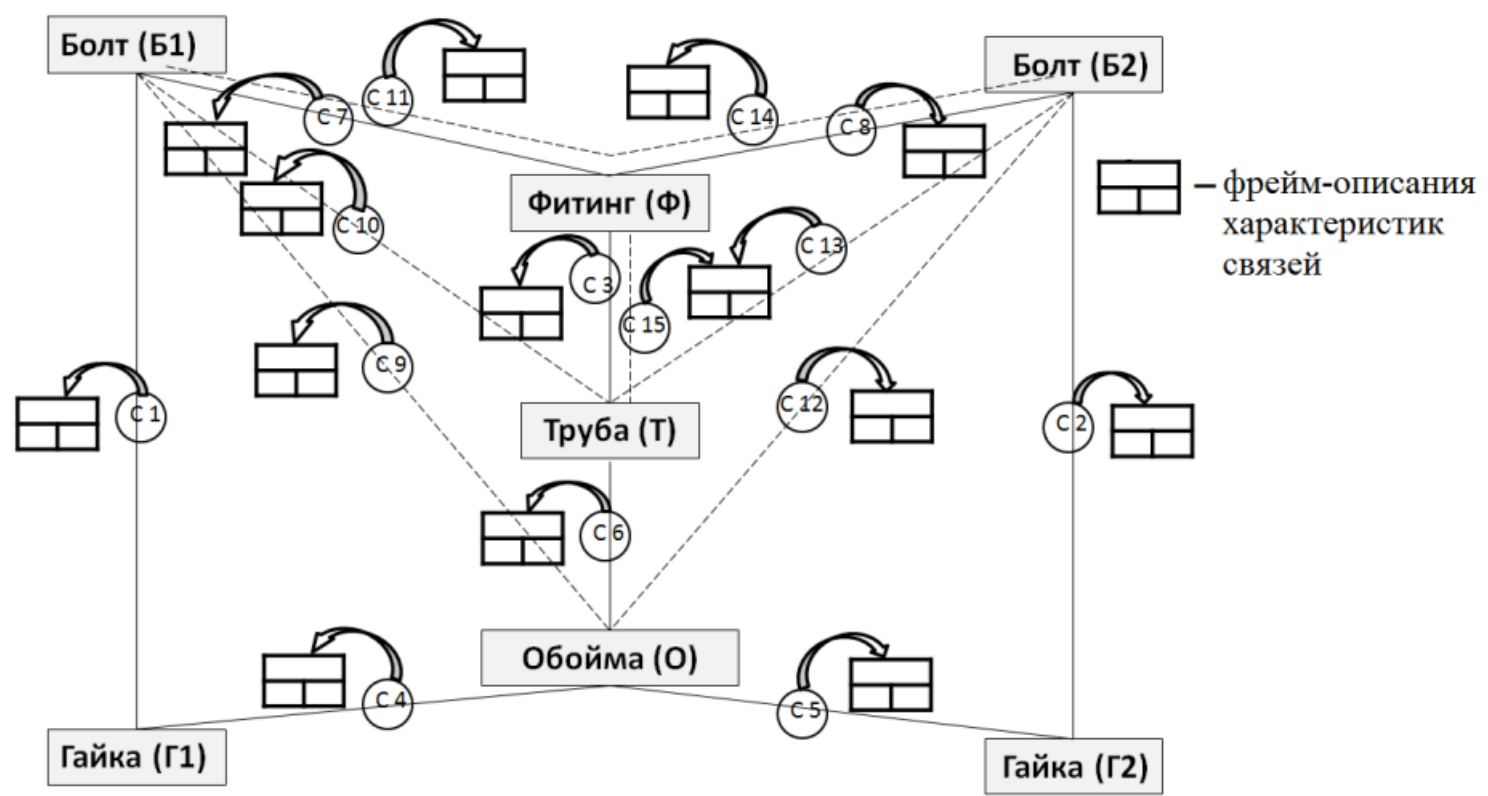

Рис. 5. Информационная модель узла соединения трубы с фитингом

Формулировки условий прочности, определяющих конструкционную целостность узла, представлены в табл. 1.

Таблица 1 - Условия прочности

\begin{tabular}{|c|c|}
\hline $\begin{array}{c}\text { Предельное } \\
\text { состояние }\end{array}$ & Формулировки условий про \\
\hline $\begin{array}{l}\text { Смятие деталей } \\
\text { преднатяжен- } \\
\text { ными болтами }\end{array}$ & $\begin{array}{l}\frac{F_{0}}{A^{b f}}<\sigma^{f}, \frac{F_{0}}{A^{s n}}<\sigma^{s}, \frac{F_{0}}{A^{s p}}<\sigma^{p}, \text { где } F_{0}-\text { усилие затяжки болта; } A^{b f}, A^{s n}, A^{s p}- \\
\text { соответственно площади контактного взаимодействия головки болта и фи- } \\
\text { тинга, обоймы и гайки, обоймы и трубы; } \sigma^{f}, \sigma^{s}, \sigma^{p}-\text { соответственно проч- } \\
\text { ность на смятие фитинга, обоймы, трубы. }\end{array}$ \\
\hline $\begin{array}{l}\text { Ослабл } \\
\text { затяжк }\end{array}$ & $\begin{array}{l}F_{0}^{\text {real }}=F_{0}^{\text {calc }} \text {, где } F_{0}^{\text {real }}, F_{0}^{\text {calc }}-\text { соответственно фактическое и расчетное (проект- } \\
\text { ное) усилие затяжки болта. }\end{array}$ \\
\hline $\begin{array}{l}\text { Разрушє } \\
\text { клеевог }\end{array}$ & $\begin{array}{l}\sigma_{g l u e}<\sigma_{g l u e_{c r}}, \text { где } \sigma_{g l u e}-\text { эквивалентные напряжения в клеевом слое; } \sigma_{g l u e_{c r}}-\text { проч- } \\
\text { ность клеевого слоя. }\end{array}$ \\
\hline $\begin{array}{l}\text { Нарушение } \\
\text { плотности } \\
\text { соединения } \\
\end{array}$ & $\begin{array}{l}F_{s t}<F_{f r}^{f p}, \text { где } F_{s t}-\text { растягивающая сила в стержне каркаса; } F_{f r}^{f p}-\text { сила трения } \\
\text { между фитингом и трубой, стянутых преднатяженными болтами. }\end{array}$ \\
\hline $\begin{array}{l}\text { Разрушение } \\
\text { обоймы }\end{array}$ & $\begin{array}{l}\sigma^{s}<\sigma_{b}^{s}, \text { где } \sigma^{s}-\text { максимальные эквивалентные напряжения в обойме; } \sigma_{b}^{s}-\text { вре- } \\
\text { менное сопротивление разрушению материала обоймы. }\end{array}$ \\
\hline $\begin{array}{l}\text { Разрушение } \\
\text { фитинга }\end{array}$ & $\begin{array}{l}\sigma^{f}<\sigma_{b}^{f}, \text { где } \sigma^{f}-\text { максимальные эквивалентные напряжения в фитинге; } \sigma_{b}^{f}-\text { вре- } \\
\text { менное сопротивление разрушению материала фитинга. }\end{array}$ \\
\hline $\begin{array}{l}\text { Разрушение } \\
\text { трубы }\end{array}$ & $\begin{array}{l}\sigma^{p}<\sigma_{b}^{p}, \text { где } \sigma^{p}-\text { максимальные эквивалентные напряжения в трубе; } \sigma_{b}^{p}-\text { времен- } \\
\text { ное сопротивление разрушению материала трубы. }\end{array}$ \\
\hline Срез болтов & $\begin{array}{l}\tau^{b}<\tau_{c u t}^{b}, \text { где } \tau^{b}-\text { максимальное касательное напряжение в сечении болта; } \\
\tau_{c u t}^{b}-\text { предел прочности материала болта на срез. }\end{array}$ \\
\hline
\end{tabular}


Нарушения рассматриваемых условий прочности происходит в различные периоды времени в течение жизненного цикла. Принципиально важно рассматривать эти нарушения как взаимосвязанные события и процессы.

Методический подход к построению информационной модели живучести, учитывающей структурные взаимодействия элементов конструкции, взаимосвязь и взаимообусловленность процессов накопления повреждений, обосновывается следующим образом. Многоуровневый процесс накопления повреждений рассматривается как информационный процесс и описывается семантической сетью каузального типа. Семантическая сеть представляет собой ориентированный граф, где вершины представляют собой понятия предметной области (в данном случае повреждения как состояния, ассоциированные с той или иной неоднородностью), а дуги - отношения между вершинами. В данном случае имеем дело с отношениями причинности. Построенная таким образом семантическая сеть представляет собой сценарий развития повреждений, связанных с теми или иными неоднородностями, вплоть до потери конструкционной целостности. Многоуровневость процесса повреждений характеризуется количеством дуг, связывающих одновременно протекающие процессы развития повреждений. Сами же отношения причинности не обязательно имеют смысл детерминированной обусловленности, а могут отражать различную степень причинной обусловленности. Рассматриваемая информационная модель соответствует пониманию многоуровневого процесса накопления повреждений как системы, в которой существует взаимовлияние и взаимообусловленность развития отдельных повреждений.

Таким образом, информационная модель живучести представляет собой семантическую сеть каузального типа, являющуюся сценарием многоуровневого процесса накопления повреждений (рис. 6). С вершинами графа, описывающими повреждения, ассоциируются конструктивные элементы, в которых эти повреждения локализованы.

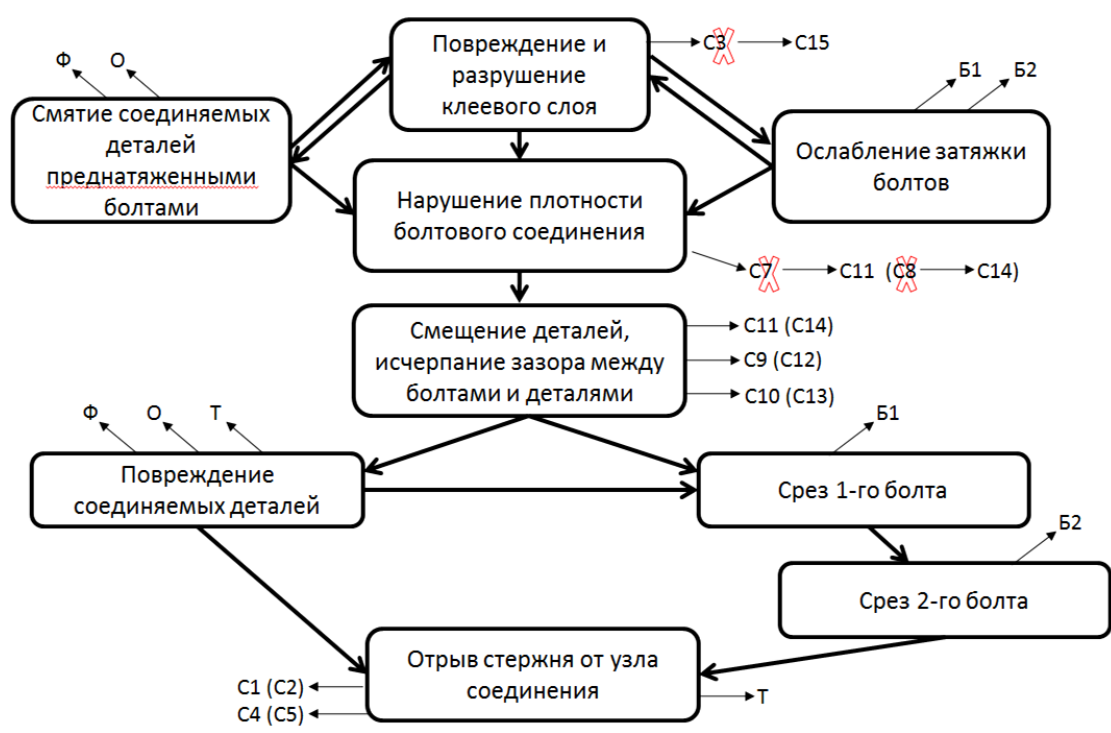

Рис. 6. Информационная модель живучести узла сочленения

Необходимость разработки и апробации человеко-машинной процедуры анализа живучести повреждаемой конструкции обусловлена тем, что реализация всей цепочки взаимосвязанных повреждений предполагает использование неформальных процедур перестройки модели и расчетной схемы.

Применительно к рассматриваемой конструкции вычислительная модель неповрежденной конструкции включает базовые разрешающие уравнения и алгоритм метода конечных элементов в перемещениях [11], а также нелинейные процедуры решения трехмерной контактной задачи теории упругости с переменной зоной контакта с трением [12]. Формули- 
ровка численной (конечно-элементной) модели узла сочленения включает два шага нагружения. На первом шаге задается предварительное натяжение болтов и определяется начальное (собственное) напряженное состояние механической системы. На втором реализуется итерационный алгоритм решения нелинейной контактной задачи.

Численная модель разработана для узла сочленения со следующими физикотехническими параметрами (рис. 7). Свойства материалов: гайка, болт, обойма - углеродистая сталь с модулем Юнга $E=210000$ МПа, коэффициентом Пуассона $v=0,3$; фитинг - тканый полимерный композит: $E_{x}=E_{y}=60500 \mathrm{MПа;} E_{z}=6900 \mathrm{MПа;} v_{x y}=0,03 ; v_{y z}=v_{x z}=0,3$; $G_{x y}=19500 \mathrm{MПа;} G_{y z}=G_{x z}=2700 \mathrm{MПа;} \mathrm{труба} \mathrm{-} \mathrm{однонаправленный} \mathrm{полимерный} \mathrm{композит:}$ $E_{x}=60900 \mathrm{MПа;} E_{y}=27000 \mathrm{MПа} ; E_{z}=15000 \mathrm{MПа} ; v_{x y}=0,07 ; v_{y z}=0,4 ; v_{x z}=0,27 ; G_{x y}=G_{x z}=$ $4700 \mathrm{MПа;} G_{y z}=3100 \mathrm{MПа;} \mathrm{коэффициент} \mathrm{трения}=0,15$.

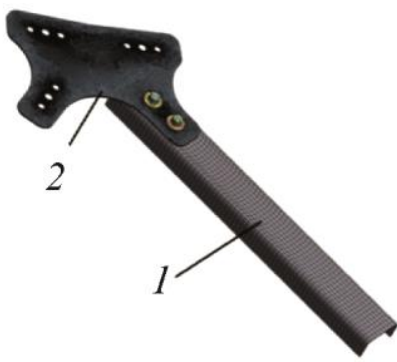

$a$

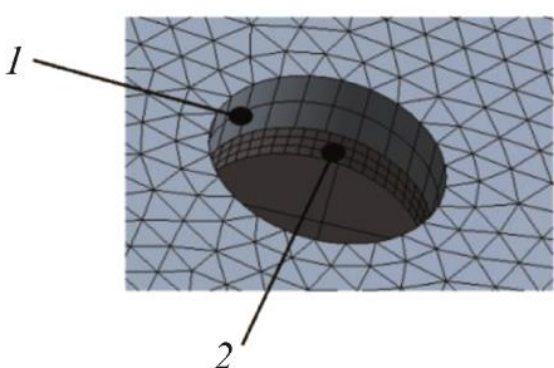

$\sigma$

Рис. 7. Конечно-элементная модель: 1 - труба; 2 - фитинг

Рассмотрим далее примеры результатов, полученных при практической работе с информационной моделью живучести.

\section{3. Результаты и обсуждение}

В неповрежденном узле труба и фитинг соединяются как клеевым слоем, так и парой преднапряженных болтов. Излишнее усилие затяжки может привести как к смятию соединяемых деталей, так и инициировать растрескивание клеевого слоя с последующим его разрушением при знакопеременном нагружении. В результате серии конечно-элементных расчетов с варьированием усилия затяжки болтов построены зависимости максимумов эквивалентных напряжений в отдельных структурных элементах от усилия затяжки болтов, аппроксимированные линейными уравнениями (рис. 8). Кроме того, получена зависимость максимальных напряжений в клеевом слое от преднатяженных болтов. Она аппроксимируется уравнением $\sigma_{\text {glue }}=0,0006 \cdot F_{0}$.

Таким образом, на этом шаге установлено, что прежде всего смятие может возникнуть в трубе, именно с этого структурного элемента инициируется развитие повреждений.

Находясь в условиях неопределенности относительно прочности композитного материала на смятие, сформируем по литературным данным интервал возможных значений предела прочности на смятие для аналогичных материалов. Этот интервал составляет [159...600] МПа [13-15]. Примем для дальнейших рассуждений минимальное значение $-\sigma^{p}=159$ МПа. Прочность клеевого слоя по экспериментальным данным составляет $\sigma_{g l u e_{c r}}=12$ МПа. Подставляя значения прочностных характеристик композита и клеевого слоя в полученные на предыдущем шаге уравнения $159=0,0219 \cdot F_{0} ; 12=0,0006 \cdot F_{0}$, получим значения $F_{01}=7294 \mathrm{H} ; F_{02}=20000 \mathrm{H}$, при превышении которых, соответственно, инициируются процессы смятия трубы и разрушения клеевого слоя в области преднапряженных болтов (рис. 9, зона б). 


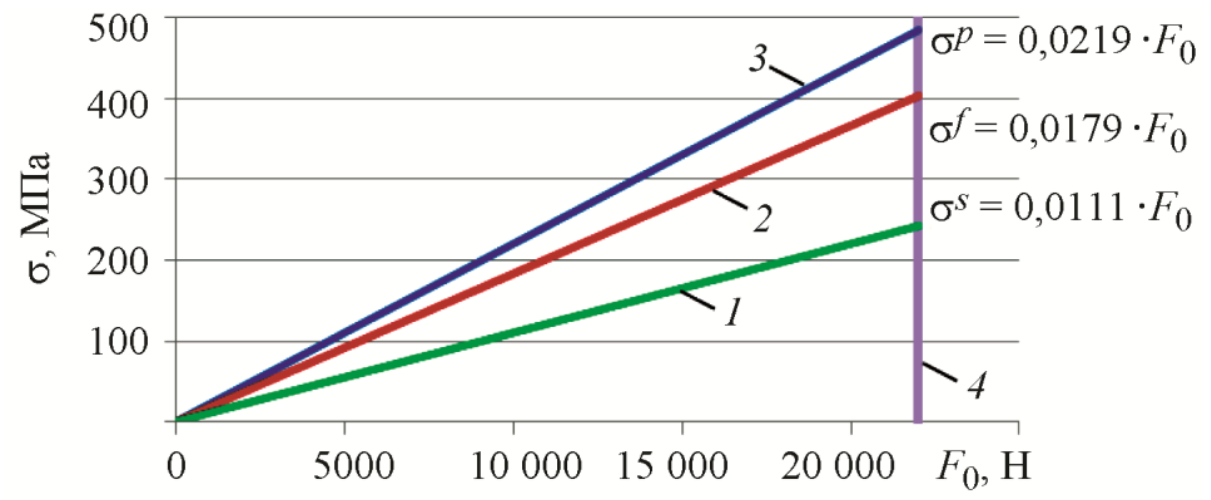

Рис. 8. Зависимость эквивалентных напряжений в структурных элементах от усилия затяжки болта: 1 - обойма; 2 - фитинг; 3 - труба; 4 - минимальное разрушающее усилие болта по ГОСТ Р 52627-2006 (ИСО 898-1:1999)

Таким образом, на этом шаге определены условия:

$$
\begin{aligned}
& F_{0}<F_{01} ; \\
& F_{0}<F_{02},
\end{aligned}
$$

нарушение которых является триггерами сценария развития повреждений, т. е. событиями, изменяющими состояние системы таким образом, что инициируются те или иные цепочки семантической сети-информационной модели живучести.

При совместном действии усилия затяжки болтов $F_{0}$ и растягивающей силы $F_{s t}$ опасности повреждения клеевого слоя зависят от соотношения $F_{0}$ и $F_{s t}$. Установлено, что при значении растягивающей силы $F_{s t} \geq 5400 \mathrm{H}$ вне зависимости от усилия затяжки возникают условия дополнительного повреждения клеевого слоя по его краям (рис. 9, зона в), т. е. в области кромок соединяемых деталей (рис. 10$): 12=4 \mathrm{e}-7 \cdot F_{s t}^{2}+0,0002 \cdot F_{s t}$. Таким образом, определено еще одно неравенство:

$$
F_{s t}<5400 \mathrm{H}
$$

нарушение которого является триггером сценария развития повреждений.

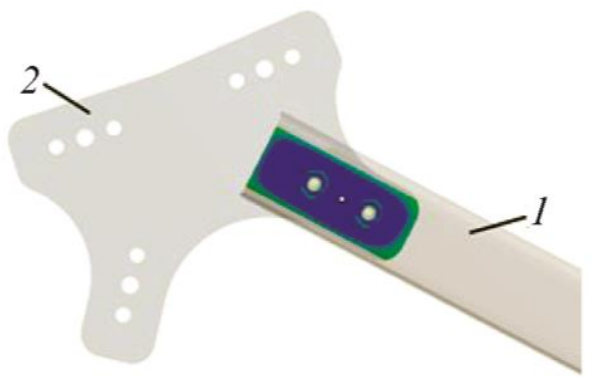

$a$

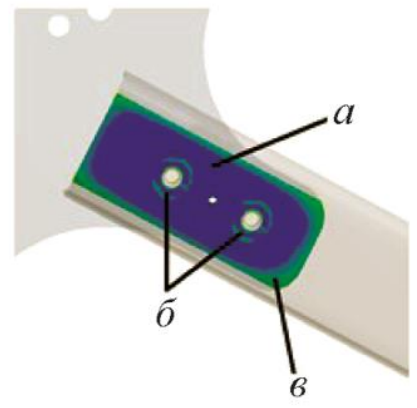

$\sigma$

Рис. 9. Зоны повреждения клеевого слоя: 1 - труба; 2 - фитинг; $a$ - клеевой слой; $\sigma$ - зона повреждения клеевого слоя преднатяженными болтами; в - зона повреждения клеевого слоя растягивающей силой

Рассмотрение условий (1)-(3) с учетом возможности их совместного осуществления приводит к пяти вариантам поврежденного состояния узла сочленения (табл. 2; рис. 9). Один 
из этих вариантов или ослабление затяжки болтов в соответствии с рис. 6 в конечном итоге приведет к нарушению плотности болтового соединения.

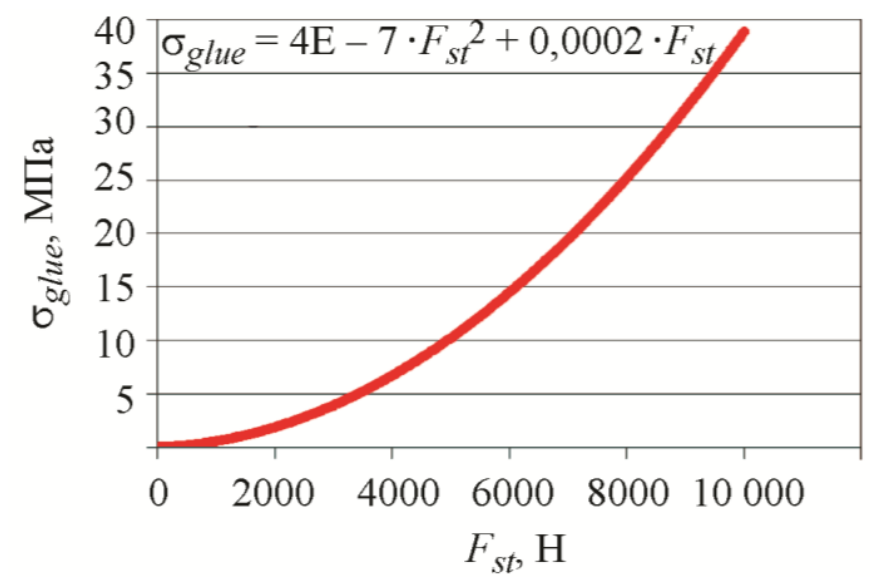

Рис. 10. Зависимость напряжений по краям клеевого слоя (рис. 9, зона в) от величины растягивающей силы

Таблица 2 - Варианты поврежденных состояний узла сочленения

\begin{tabular}{|c|l|c|c|c|}
\hline \multirow{2}{*}{$№$} & \multicolumn{1}{|c|}{ Развитие повреждений } & \multicolumn{3}{|c|}{ Выполнение условий } \\
\cline { 3 - 5 } & \multicolumn{1}{|c|}{$(1)$} & $(2)$ & Да \\
\hline 1 & Смятие трубы, клеевой слой неповрежден & Нет & Да \\
\hline 2 & Повреждение клеевого слоя по его краям & Да & Да & Да \\
\hline 3 & $\begin{array}{l}\text { Смятие трубы и повреждение клеевого слоя } \\
\text { преднатяженными болтами }\end{array}$ & Нет & Нет & Нет \\
\hline 4 & $\begin{array}{l}\text { Смятие трубы и повреждение клеевого слоя по } \\
\text { его краям }\end{array}$ & Нет & Да Нет & Нет \\
\hline 5 & $\begin{array}{l}\text { Смятие трубы и повреждение клеевого слоя } \\
\text { преднатяженными болтами и повреждение } \\
\text { клеевого слоя по его краям }\end{array}$ & Нет \\
\hline
\end{tabular}

Дальнейший шаг анализа (реализация состояния «смещение деталей, исчерпание зазора между болтами и деталями» в информационной модели живучести на рис. 6) требует трансформации трехмерной геометрии модели, заключающейся в смещении трубы и фитинга в противоположные стороны относительно болтов на величину зазора. Далее выполняются вычислительные процедуры в соответствии с рис. 6 до полной потери конструкционной целостности узла.

\section{4. Заключение}

Предложен и апробирован междисциплинарный методический подход к построению и анализу живучести структурно-сложных и структурно-неоднородных конструкций. Он заключается в разработке и использовании структурной и информационной моделей конструкции, на основе которых разрабатывается информационная модель живучести - сценарий многоуровневого процесса накопления повреждений. Исследование модели живучести с использованием аналитических, численных, экспертных методов позволяет описать все возможные состояния конструкции в течение жизненного цикла с последующей оценкой соответствующих рисков. 
Апробация подходов на примере узла сочленения стержней каркаса крупногабаритного прецизионного рефлектора зеркальной антенны позволила установить критические значения внутренних и внешних воздействий (усилия преднатяжения болтов и растягивающих усилий в стержнях каркаса), соответствующие реализации разных вариантов развития повреждений.

\section{Литература}

1. Frolov K. V. Kudinov V. M., Makhutov N. A. Applications of Mechanics to Technological Problems (Mechanical Engineering \& Applied Mechanics). - CRC Press, 1999. - 410 p.

2. $\quad$ Case J., Chilver A. H., Ross C. T. F. Strength of Materials and Structures / 4th edition. Butterworth-Heinemann, 1999. - P. 724.

3. Jenkins C. H. M. Mechanics of materials. A Modern Integration of Mechanics and Materials in Structural Design. - Elsevier Academic Press, 2005. - 408 p.

4. Parnes R. Solid Mechanics in Engineering / first edition. - John Wiley \& Sons, 2001. $748 \mathrm{p}$.

5. Popov E. P. Engineering Mechanics of Solids / second edition. - Prentice Hall of India, 1998. -888 p.

6. Gol'denblat I. I. Some Problems of the Mechanics of Deformable Media, Noordhoff, 1962. $304 \mathrm{p}$.

7. Перельмутер А. В. О расчетах на прогрессирующее обрушение // Вестник МГСУ. 2008. - № 1. - С. 119-129.

8. Живучесть зданий и сооружений при запроектных воздействиях / В. И. Колчунов, Н. В. Клюева, Н. Б. Андросова, А. С. Бухтиярова. - М. : Издательство АСВ, 2014. - 208 с.

9. Расчет динамических усилий в конструктивно-нелинейных элементах стержневых пространственных систем при внезапных структурных изменениях / В. А. Гордон, Н. В. Клюева, Т. В. Потураева, А. С. Бухтиярова // Строительная механика и расчет сооружений. - 2008. - № 6. - С. 26-30.

10. Махутов Н. А., Петров В. П., Резников Д. О. Оценка живучести сложных технических систем // Проблемы безопасности и чрезвычайных ситуаций. - 2009. - № 3. - С. 47-66.

11. Bathe K.-J. Finite element procedures. - New Jersey : Prentice-Hall, 1996. - 1038 p.

12. Wriggers P. Computational contact mechanics. - Berlin-Helderberg : Springer, 2006. - 521 p.

13. Комаров Г. В. Свойства ПКМ, влияющие на их способность соединяться // Полимерные материалы. - 2010. - № 2-3. - С.18-27.

14. Двейрин А. 3., Кривенда С. П. Испытание на смятие слоистых пластиков // Вопросы проектирования и производства конструкций летательных аппаратов: сб. науч. трудов. Нац. аэрокосм. ун-та им. Н.Е. Жуковского «ХАИ». - 2011. - Вып. 1 (65). - С. 20-28.

15. Семин М. И. Расчеты соединений элементов конструкций из полимерных материалов на прочность и долговечность: монография. - М. : МАДИ, 2016. - 92 с. 